\title{
Functional Significance of Medial Olivocochlear System Morphology in the Mouse Cochlea
}

\author{
So Young Park · Jung Mee Park · Sang A Back · Sang Won Yeo · Shi Nae Park \\ Department of Otorhinolaryngology-Head and Neck Surgery, College of Medicine, The Catholic University of Korea, Seoul, Korea
}

Objectives. Baso-apical gradients exist in various cochlear structures including medial olivocochlear (MOC) efferent system. This study investigated the cochlear regional differentials in the function and morphology of the MOC system, and addressed the functional implications of regional MOC efferent terminals (ETs) in the mouse cochlea.

Methods. In CBA/J mice, MOC reflex (MOCR) was assessed based on the distortion product otoacoustic emission in the absence and presence of contralateral acoustic stimulation. High, middle, and low frequencies were grouped according to a mouse place-frequency map. Cochlear whole mounts were immunostained for ETs with anti- $\alpha$-synuclein and examined using confocal laser scanning microscopy. The diameters of ETs and the number of ETs per outer hair cell were measured from the z-stack images of the basal, middle and apical regions, respectively.

Results. The middle cochlear region expressed large, clustered MOC ETs with strong MOCR, the base expressed small, less clustered ETs with strong MOCR, and the apex expressed large, but less clustered ETs with weak MOCR.

Conclusion. The mouse cochlea demonstrated regional differentials in the function and morphology of the MOC system. Strong MOCR along with superior MOC morphology in the middle region may contribute to 'signal detection in noise,' the primary efferent function, in the best hearing frequencies. Strong MOCR in spite of inferior MOC morphology in the base may reflect the importance of 'protection from noise trauma' in the high frequencies.

Keywords. Baso-Apical Gradient; Contralateral Suppression; Distortion Product Otoacoustic Emission; Efferent Terminal; Medial Olivocochlear Reflex

\section{INTRODUCTION}

Medial olivocochlear (MOC) efferent system has two presumed roles related with cochlear mechanics in noise. The most commonly accepted is that it facilitates the detection of signals in background noise by attenuating the gain of the cochlear amplifier to physiological stimuli [1-3]. Efferent fibers have also been documented to protect the ear against acoustic injury. Experi-

\footnotetext{
- Received March 31, 2016

Revised June 7, 2016

Accepted June 19, 2016

- Corresponding author: Shi Nae Park

Department of Otorhinolaryngology-Head and Neck Surgery, Seoul St.

Mary's Hospital, College of Medicine, The Catholic University of Korea,

222 Banpo-daero, Seocho-gu, Seoul 06591, Korea

Tel: +82-2-2258-6215, Fax: +82-2-595-1354

E-mail: snparkmd@catholic.ac.kr
}

mental studies proposed that the strength of the MOC reflex (MOCR) may be the indicator of noise susceptibility [4]. MOC neurons that originate in the medial portion of the superior olive and project to the outer hair cells (OHCs) constitute the efferent arm of the MOCR pathway [5,6]. MOC effects are reflected in changes of otoacoustic emissions (OAEs) in the presence of ipsilateral and/or contralateral acoustic stimulation (CAS). Distortion-product OAE (DPOAE)-based contralateral MOCR provides the test of choice for the integrity and strength of the MOC system $[2,6]$.

Baso-apical gradients exist in various cochlear structures along the length of the cochlea $[7,8]$. It is also well known that the cochlea has a spatial gradient in the susceptibility to noise with the most vulnerable OHCs at the basal end [9]. In addition, MOC neurons demonstrate sharp frequency tuning curves with tonotopicity that is found consistently within the auditory path-

Copyright @ 2017 by Korean Society of Otorhinolaryngology-Head and Neck Surgery.

This is an open-access article distributed under the terms of the Creative Commons Attribution Non-Commercial License (http://creativecommons.org/licenses/by-nc/4.0)

which permits unrestricted non-commercial use, distribution, and reproduction in any medium, provided the original work is properly cited. 
ways $[5,10]$. Maison et al. [11,12] have reported longitudinal gradients of MOC innervation density and the strength of efferent effects along the cochlear spiral in the mouse. This study aimed to confirm the cochlear regional differentials in the function and morphology of the MOC system and to address the functional implications of the regional MOC efferent terminals (ETs) in the mouse cochlea.

\section{MATERIALS AND METHODS}

Four-week-old male CBA/J mice were purchased from Orient Bio (Sungnam, Korea) and kept in our animal facility. Experiments were performed on both ears in 8 mice. All animal procedures followed the national ethical guidelines and relevant laws. The experimental protocol was approved by the Animal Care and Use Committee of the Catholic University of Korea College of Medicine.

\section{Auditory tests}

Mice were anesthetized with intraperitoneal injection of a mixture of zolazepam-tiletamine $(5 \mathrm{mg} / \mathrm{kg})$ and xylazine $(5 \mathrm{mg} / \mathrm{kg})$. All tests were conducted in a custom-made sound-attenuated chamber for mice. For hearing screening, auditory brainstem responses (ABRs) to click $(0.1 \mathrm{msec}, 19.3 / \mathrm{sec})$ and tone bursts $(8 / 16 / 32 \mathrm{kHz}, 1.5 \mathrm{msec}, 21.1 / \mathrm{sec})$ were recorded using SmartEP fitted with high-frequency transducers and high-frequency software ver. 2.33 (Intelligent Hearing Systems, Miami, FL, USA). Test stimuli were delivered to the ear canal through a miniature insert earphone from $90 \mathrm{~dB}$ to $10 \mathrm{~dB}$ sound pressure level (SPL) in $5 \mathrm{~dB}$ steps. Electrical potentials in the first $12 \mathrm{msec}$ after the stimuli were sampled via subdermal stainless steel needle electrodes at the vertex (active) and pinna (reference) with 0.1-3 $\mathrm{kHz}$ bandpass filtering, and averaged for 256 sweeps. Thresholds were defined as the lowest stimulus intensity where the typical wave pattern was still identified. SmartOAE ver. 4.26 (Intelligent Hearing Systems) was used to measure the $2 f_{1}-f_{2}$ DPOAE in the ear canal and to obtain DP-gram that displays DPOAE amplitude plotted as a function of $f_{2}$ frequency. DPOAE recordings

\section{H I}

- The mouse cochlea demonstrated regional differentials in the function and morphology of the medial olivocochlear (MOC) system.

- The middle cochlear region expressed large, clustered MOC terminals with strong MOC reflex (MOCR) in mice.

- The base expressed small, less clustered MOC terminals with strong MOCR.

- The apex expressed large, but less clustered MOC terminals with weak MOCR. were made for $f_{2}$ frequencies from 6.5 to $35 \mathrm{kHz}$ at 8 points per octave using primary tone paradigm set as follows: $L_{1}=65 \mathrm{~dB}$, $L_{2}=55 \mathrm{~dB}$ SPL, and $f_{1} / f_{2}=1.22$. Stimulus signals were passed through ER-10B+ probe microphone inserted into the ear canal in conjunction with two types of transducers: ER-2 transducer (Etymotic Research, Elk Grove Village, IL, USA) for frequencies from 6 to $16 \mathrm{kHz}$ and high-frequency transducer (Intelligent Hearing Systems) for frequencies from 16 to $32 \mathrm{kHz}$. The output was sampled at $128 \mathrm{kHz}$ using a 16-bit A/D converter. Four blocks were acquired in total and each block consisted of 32 sweeps. Tests were performed under two stimulus conditions of the opposite ear with and without CAS. Broadband noise MOC elicitor was presented to the contralateral ear at $55 \mathrm{~dB}$ SPL that does not evoke stapedial reflex in mammals [13].

\section{Immunostaining and quantification of MOC terminals}

Mice were anesthetized and killed to harvest the cochleas, which were processed for whole mount immunofluorescent staining and confocal microscopy. The cochlea was perfused and fixed with $2 \%$ paraformaldehyde, and then decalcified in ethylenediaminetetraacetic acid (EDTA) for 2-4 days. The lateral wall, Reissner's membrane, and tectorial membrane were removed in order after the otic capsule was opened. In whole mount cochleas, the ETs were made visible by immunofluorescent staining for $\alpha$-synuclein, an efferent synaptic vesicular protein. Specimens were blocked in 5\% normal goat serum and 0.3\% Triton X-100 for 1 hour, and incubated with 1:100 anti- $\alpha$ synuclein antibody (purified mouse anti- $\alpha$-synuclein, BD Biosciences, San Jose, CA, USA) at $4^{\circ} \mathrm{C}$ overnight in a humid chamber. They were rinsed and incubated with 1:4,000 fluorescent secondary antibody (Alexa Fluor 555 goat anti-mouse IgG, Thermo Fisher Scientific, Waltham, MA, USA) for 2 hours. After the secondary antibodies had been removed, specimens were exposed to Vectashield with DAPI (Vector Laboratories, Burlingame, CA, USA) which allows visualization of the $\mathrm{OHC}$ nuclei. The frequency regions of interest were localized along the cochlear spiral at a low magnification according to a place-frequency map of mouse cochlea published previously [14]. Eighteen percent distance from the apex designated $8 \mathrm{kHz}$ region, $43 \%$ distance designated $16 \mathrm{kHz}$, and $68 \%$ distance designated $32 \mathrm{kHz}$. Stained whole mounts were examined by confocal laser scanning microscopy (LSM 510 Meta, Carl Zeiss, Jena, Germany). As the ET-OHC synapses are located at different heights, a confocal z-stack was obtained from each region of the cochlea. Approximately 30 series of images were acquired per a region by focusing from top to bottom with a $0.6 \mu \mathrm{m}$ step in the $\mathrm{z}$-axis through the whole mount specimen under a magnification of 1,000. The acquired z-plane images were reconstructed using IPLab software (BioVision Technologies Inc., Exton, PA, USA) to create a single $z$-stack image. The captured images were imported into Image J software (National Institutes of Health, Bethesda, MD, USA). For quantitative morphometric analysis, 
two indices were used: the ET diameter and the number of ETs per OHC. The diameters of all visible ETs in a single image were measured and averaged. The number of all ETs (range, 44 to 91) was counted and divided by the number of OHCs (range, 31 to 39) shown in that image.

\section{Data processing and statistical analysis}

DPOAE amplitude was measured from the noise floor at each test frequency. Data points of an amplitude less than $6 \mathrm{~dB}$ were rejected. A DPOAE change by the MOC effect was defined as CAS-on amplitude minus baseline amplitude. Negative changes indicated suppression and positive changes, enhancement. The DPOAE change was normalized to the baseline amplitude and termed suppression ratio as the measure of MOCR strength at a valid data point. $F_{2}$ frequencies were grouped into three ranges based on the same mouse place-frequency map: the low frequencies (LF) included 6,573-10,779 Hz, which corresponds approximately to a $<30$ percent distance region from the apex; the middle frequencies (MF), 11,904-23,592 Hz, a 30-60 percent distance region; the high frequencies (HF), 26,048-35,056 $\mathrm{kHz}, \mathrm{a}>60$ percent distance region. Within each frequency range, the suppression ratios at all data points from 16 ears were averaged. Statistical analysis was done using SPSS ver. 18.0 (SPSS Inc., Chicago, IL, USA) at a two-tailed significance level of 0.05. Two way ANOVA was performed in advance of every statistical analysis to rule out any interaction between the group and the side of the tested ears. The suppression ratios were compared across the three frequency ranges. The morphometric indices of the ETs were compared across the three regions of the cochlea. One way ANOVA and post hoc Tukey's test were performed after Shapiro-Wilk test for normal distribution and Levene's test for the equality of variances. Otherwise, Kruskal-Wallis test and post hoc Mann-Whitney $U$-test were applied with Bonferroni's correction.

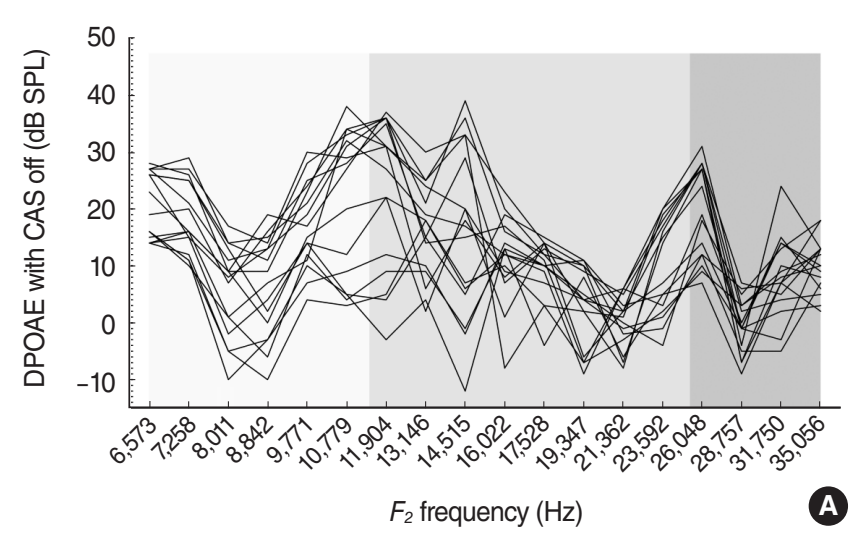

\section{RESULTS}

\section{DPOAE-based MOCR activity}

ABR thresholds at click, 8, 16, and $32 \mathrm{kHz}$ tone bursts were $16.3 \pm 0.6$ (mean \pm standard error of mean [SE]), $16.3 \pm 0.6$, $18.4 \pm 0.9$, and $19.4 \pm 0.8 \mathrm{~dB}$ SPL respectively. All experimental mice had good hearing with ABR thresholds less than $20 \mathrm{~dB}$ SPL at the test stimuli on both ears. DP-grams obtained from 16 ears displayed a consistent pattern of amplitude variations with alternating peaks and dips in quiet, and in the presence of CAS (Fig. 1). There were no statistical differences in DPOAE levels between the right and left ears: $12.4 \pm 7.7 \mathrm{~dB}$ SPL for the right and 11.8 $\pm 7.5 \mathrm{~dB}$ SPL for the left ears (grand mean of the mean DPOAEs at test frequencies \pm standard deviation). DPOAE amplitudes were in the range of -12 to $39 \mathrm{~dB}$ SPL without CAS, and -14 to $38 \mathrm{~dB}$ SPL with CAS. Valid data points for CAS were 204 (71\%) out of 288 (18 frequencies in 16 ears): 78, 84, and 42 from LF, MF, and HF respectively. CAS-evoked DPOAE changes exhibited both suppressions and enhancements ranged from -16 to $11 \mathrm{~dB}$ SPL (Fig. 2A). Mean (SE) suppression ratio in LF/MF/HF was $0.19(0.02) / 0.39(0.06) / 0.42$ (0.09) from $39 / 49 / 24$ suppression points. Significant differences were shown between LF and MF $(P=0.012)$, and between LF and HF $(P=$ 0.005) (Fig. 2B).

\section{Morphometry of MOC efferent terminals}

Whole mount organ of Corti labeled with anti- $\alpha$-synuclein showed clustered MOC ETs in the OHC area (Fig. 3A). Mean (SE) diameters of MOC terminals were 1.70 (0.04), 1.84 (0.04), and $1.53(0.06) \mu \mathrm{m}$ in the 8,16 , and $32 \mathrm{kHz}$ regions respectively with significant differences between the 16 and $32 \mathrm{kHz}$ $(P<0.001)$, and between the 8 and $32 \mathrm{kHz}$ regions $(P=0.048)$ (Fig. 3B). Mean (SE) numbers of MOC terminals per OHC were 1.7 (0.06), 2.3 (0.04), and $1.8(0.03)$ in the 8, 16, and $32 \mathrm{kHz}$ regions respectively with significant differences between the 8

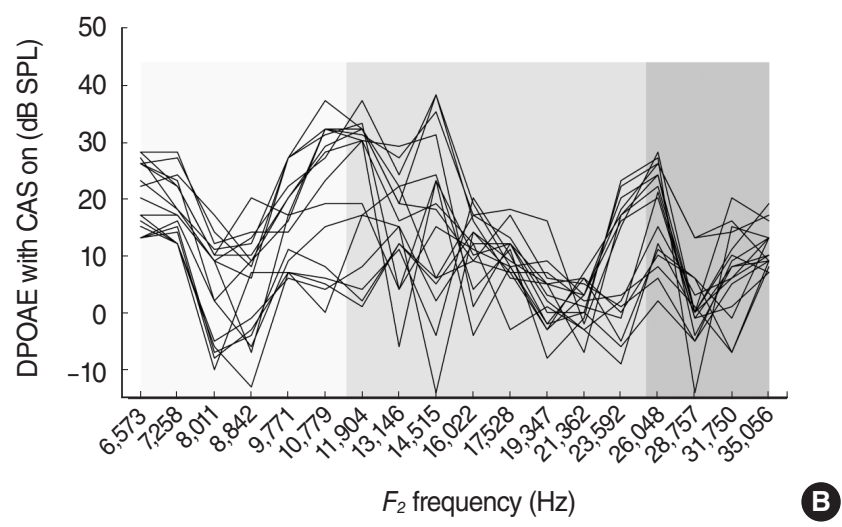

Fig. 1. Individual DP-grams of 16 ears at $65 \mathrm{~dB}$ sound pressure level (SPL) primary tone level (A) in quiet, and (B) with contralateral acoustic stimulation (CAS) showing alternating peaks and dips. DPOAE, distortion-product otoacoustic emission. 
and $16 \mathrm{kHz}(P<0.001)$, and between the 16 and $32 \mathrm{kHz}$ regions $(P<0.001)$ (Fig. 3C).

\section{DISCUSSION}

We performed DPOAE-based MOCR test and microscopic quantification of the MOC terminals in mice to investigate the

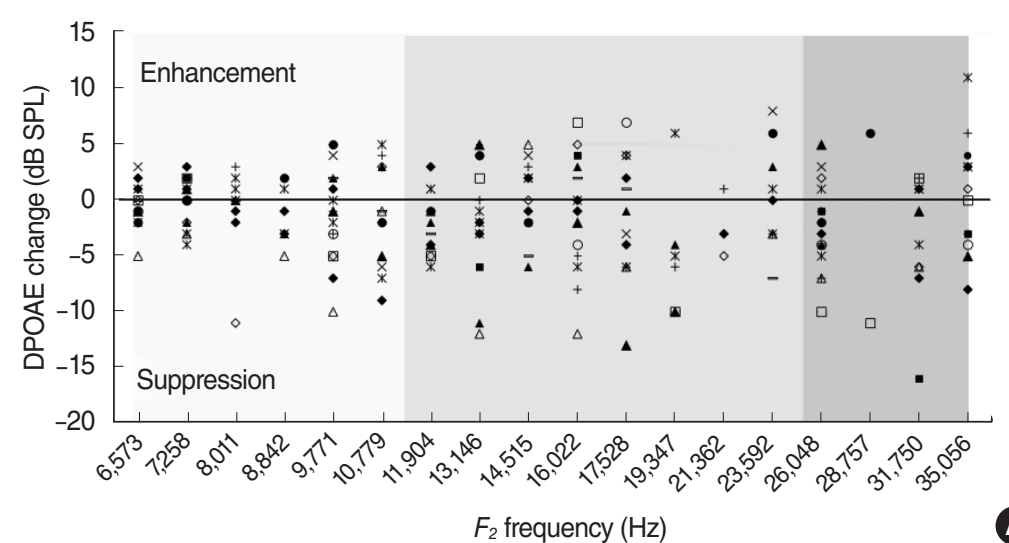

A

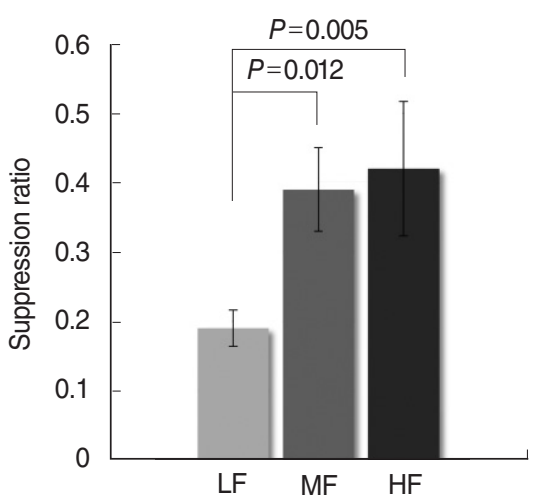

Fig. 2. (A) Plotting of distortion-product otoacoustic emission (DPOAE) changes by contralateral MOC reflex at all valid data points. Negative values indicate suppression and positive values, enhancement. (B) Comparison of DPOAE suppression ratios (suppression amplitude/baseline DPOAE) across three frequency ranges. The low frequencies (LF) covers 6,573-10,779 Hz; middle frequencies (MF), 11,904-23,592 Hz; high frequencies (HF), 26,048-35,056 Hz. Error bars indicate standard error of mean. SPL, sound pressure level.
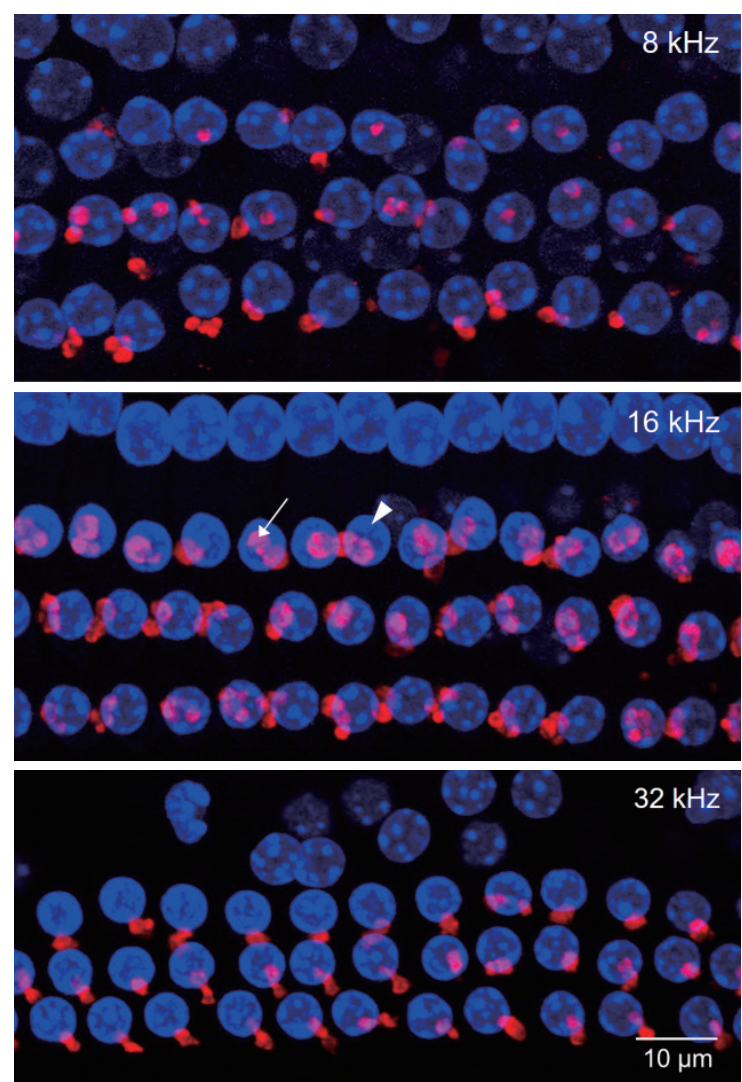

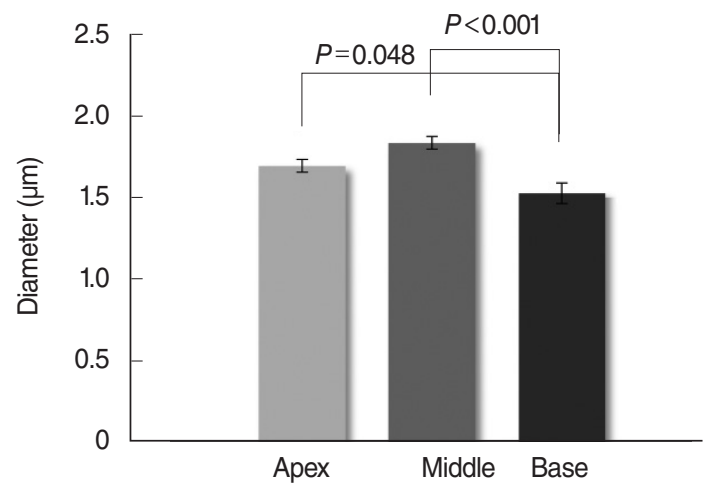

B

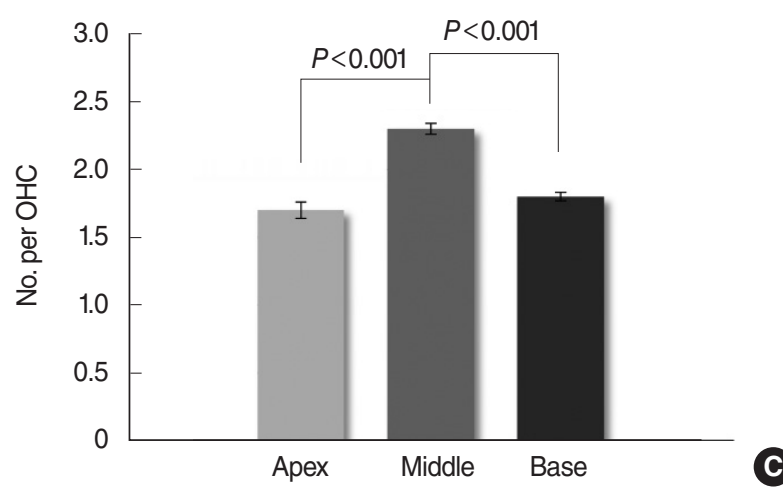

Fig. 3. (A) Representative confocal z-stacks from three cochlear regions of 8, 16, and $32 \mathrm{kHz}$, which reveal the clusters of medial olivocochlear (MOC) efferent terminals immunolabeled for a-synuclein (arrow) under the outer hair cell (OHC) nuclei stained with DAPI (arrowhead; Vectashield) $(\times 1,000)$. (B) The diameters of efferent terminals and $(C)$ the number of terminals per OHC were compared among three cochlear regions. Apex, middle, and base correspond to 8,16 , and $32 \mathrm{kHz}$ regions, respectively $(n=16)$. Error bars indicate standard error of mean. 
regional differentials in MOC function and morphology along the cochlea and to identify the relationship between them. The DP-grams at 8 points/octave showed DPOAE fine structures. Contralateral MOCR resulted in both suppressions and enhancements of DPOAEs, but suppressions occurred more frequently and exhibited larger amplitudes, as reported in the previous literatures $[15,16]$. The DPOAE is a mixed emission generated by two different mechanisms in the cochlea: nonlinear distortion from $f_{2}$ location and linear reflection from $2 f_{1}-f_{2}$ location. The vector combination of distortion and reflection components gives rise to DPOAE fine structure $[17,18]$. However, phase interference between two components can confound the interpretation of MOC effects on DPOAE: the out-of-phase cancellation at a fine-structure dip is abruptly released by contralateral noise through the phase shift in the reflection component, producing an increase in DPOAE level [19]. Therefore, we limited the index of MOCR activity to 'suppression' only in order to reject possible artifact 'enhancement' of DPOAE.

The size and number of the MOC terminals were the most remarkable in the $16 \mathrm{kHz}$ region, which was similar with the previous studies. Maison et al. [11] have documented that longitudinal gradients of MOC terminals and the suppression of compound action potential peaked in the middle of the cochlear spiral near the $10 \mathrm{kHz}$ region in the mouse, which indicates the correlation between the function and morphology of the MOC system. They have also reported that the average strength of shock-evoked DPOAE suppression was greatest in the middle $(11-22 \mathrm{kHz})$ of the test tones used $(5.6-45.2 \mathrm{kHz}$ in half-octave steps) [12]. In our study, contralateral sound-evoked DPOAE suppression ratios measured at 8 frequencies/octave were significantly larger in MF (11.9-23.6 kHz) and HF (26.0-35.1 kHz) than in LF (6.6$10.8 \mathrm{kHz}$ ) without difference between MF and HF.

Mice possess short cochleas and hear tones from 1 to near $100 \mathrm{kHz}$. The upper frequency limit for human hearing is approximately $20 \mathrm{kHz}$. The highest test frequency at $32 \mathrm{kHz}$ region in mouse cochlea corresponds roughly to $8 \mathrm{kHz}$ region in human cochlea [20]. Muller et al. [21] have documented that mice have a hearing differential across the audible range with the best $\mathrm{ABR}$ thresholds at a middle frequency band from 11 to $16 \mathrm{kHz}$. Also in humans, the middle frequencies from 0.5 to 2 $\mathrm{kHz}$ contribute mainly to speech perception. In our data, most of the DPOAE peaks were in the range of 10.8 to $14.5 \mathrm{kHz}$. It is notable that the best hearing frequencies in mice coincided approximately with the best frequencies for DPOAEs, MOCR, and the MOC ETs.

Overall, the middle cochlear region expressed large, clustered MOC ETs with strong MOCR, the base expressed small, less clustered MOC ETs with strong MOCR, and the apex expressed large, but less clustered MOC ETs with weak MOCR in mice. The base showed no correlation between the function and morphology of the MOC system. Weak MOCR with small number of MOC ETs in the apex may be related with the fact that mice perform poorly in the low frequencies [8]. However, strong MOCR in spite of inferior MOC morphology in the cochlear base may indicate the demand for 'protection from noise trauma' in the high frequencies. The superior MOC activity and morphology of the middle region may indicate the weight of 'signal detection in noise' in the best hearing frequencies or another unknown efferent function beyond MOCR.

In conclusion, the mouse cochlea demonstrated regional differentials in the function and morphology of the MOC system. Strong MOCR along with superior MOC morphology in the middle frequency region may contribute to 'signal detection in noise,' the primary efferent function, in the best hearing frequencies. Strong MOCR in spite of inferior MOC morphology in the base may reflect the importance of 'efferent protection from noise trauma' in the high frequencies.

\section{CONFLICT OF INTEREST}

No potential conflict of interest relevant to this article was reported.

\section{ACKNOWLEDGMENTS}

This work was supported in part by grants from the Basic Science Research Program of the National Research Foundation of Korea funded by the Ministry of Education, Science, and Technology (NRF-2010-0004744 and NRF-2013-R1A1A2011870).

\section{REFERENCES}

1. Christopher Kirk E, Smith DW. Protection from acoustic trauma is not a primary function of the medial olivocochlear efferent system. J Assoc Res Otolaryngol. 2003 Dec;4(4):445-65.

2. Rabbitt RD, Brownell WE. Efferent modulation of hair cell function. Curr Opin Otolaryngol Head Neck Surg. 2011 Oct;19(5):376-81.

3. Smith DW, Keil A. The biological role of the medial olivocochlear efferents in hearing: separating evolved function from exaptation. Front Syst Neurosci. 2015 Feb;9:12.

4. Maison SF, Liberman MC. Predicting vulnerability to acoustic injury with a noninvasive assay of olivocochlear reflex strength. J Neurosci. 2000 Jun;20(12):4701-7.

5. Brown MC, de Venecia RK, Guinan JJ Jr. Responses of medial olivocochlear neurons: specifying the central pathways of the medial olivocochlear reflex. Exp Brain Res. 2003 Dec;153(4):491-8.

6. Guinan JJ Jr. Olivocochlear efferents: anatomy, physiology, function, and the measurement of efferent effects in humans. Ear Hear. 2006 Dec;27(6):589-607.

7. Mann ZF, Kelley MW. Development of tonotopy in the auditory periphery. Hear Res. 2011 Jun;276(1-2):2-15.

8. Vater M, Kossl M. Comparative aspects of cochlear functional organization in mammals. Hear Res. 2011 Mar;273(1-2):89-99.

9. Henderson D, Bielefeld EC, Harris KC, Hu BH.The role of oxidative stress in noise-induced hearing loss. Ear Hear. 2006 Feb;27(1):1-19. 
10. Guinan JJ Jr. Cochlear efferent innervation and function. Curr Opin Otolaryngol Head Neck Surg. 2010 Oct;18(5):447-53.

11. Maison SF, Adams JC, Liberman MC. Olivocochlear innervation in the mouse: immunocytochemical maps, crossed versus uncrossed contributions, and transmitter colocalization. J Comp Neurol. 2003 Jan;455(3):406-16.

12. Maison SF, Rosahl TW, Homanics GE, Liberman MC. Functional role of GABAergic innervation of the cochlea: phenotypic analysis of mice lacking GABA(A) receptor subunits alpha 1, alpha 2, alpha 5, alpha 6, beta 2, beta 3, or delta. J Neurosci. 2006 Oct;26(40):103 15-26.

13. Zhu X,Vasilyeva ON, Kim S, Jacobson M, Romney J, Waterman MS, et al.Auditory efferent feedback system deficits precede age-related hearing loss: contralateral suppression of otoacoustic emissions in mice. J Comp Neurol. 2007 Aug;503(5):593-604.

14. Viberg A, Canlon B. The guide to plotting a cochleogram. Hear Res. 2004 Nov;197(1-2):1-10.

15. Zhang F, Boettcher FA, Sun XM. Contralateral suppression of distortion product otoacoustic emissions: effect of the primary frequency in Dpgrams. Int J Audiol. 2007 Apr;46(4):187-95.
16. Wagner W, Heppelmann G, Muller J, Janssen T, Zenner HP. Olivocochlear reflex effect on human distortion product otoacoustic emissions is largest at frequencies with distinct fine structure dips. Hear Res. 2007 Jan;223(1-2):83-92.

17. Shaffer LA, Withnell RH, Dhar S, Lilly DJ, Goodman SS, Harmon KM. Sources and mechanisms of DPOAE generation: implications for the prediction of auditory sensitivity. Ear Hear. 2003 Oct;24(5): 367-79.

18. Shera CA. Mechanisms of mammalian otoacoustic emission and their implications for the clinical utility of otoacoustic emissions. Ear Hear. 2004 Apr;25(2):86-97.

19. Mishra SK, Abdala C. Stability of the medial olivocochlear reflex as measured by distortion product otoacoustic emissions. J Speech Lang Hear Res. 2015 Feb;58(1):122-34.

20. Willott JF. Overview of methods for assessing the mouse auditory system. Curr Protoc Neurosci. 2006 Feb;Chapter 8:Unit8.21A. http://dx.doi.org/10.1002/0471142301.ns0821as34.

21. Muller M, von Hunerbein K, Hoidis S, Smolders JW. A physiological place-frequency map of the cochlea in the CBA/J mouse. Hear Res. 2005 Apr;202(1-2):63-73. 